\title{
Wavelength control of tunable dense wavelength-division multiplexing sources by use of a Fabry-Perot etalon and a semiconductor optoelectronic diode
}

\author{
Chun-Liang Yang, San-Liang Lee, and Jingshown Wu
}

\begin{abstract}
A high-resolution tunable-wavelength controller is achieved by use of an etalon for control of wavelength drift and a semiconductor optical diode (SOD) for channel recognition. The etalon provides a stable wavelength reference, and the SOD can detect mode-hopping and incomplete-tuning problems in tuning a laser. With the help of a Fabry-Perot etalon as a precise wavelength reference, the usual concern with the temperature stability of a SOD can be relaxed at least tenfold compared with wavelength control with a single SOD. We demonstrate the feasibility of monitoring tunable lasers by using a Fabry-Perot laser diode (FPLD) or a semiconductor optical amplifier (SOA). The induced voltage of the FPLD and that of the SOA are modeled with analytic expressions that can help to optimize the operation of a SOD sensor. (C) 2004 Optical Society of America
\end{abstract}

OCIS codes: $\quad 060.2360,060.2340$.

\section{Introduction}

Tunable laser sources (TLS's) are critical components of dense wavelength-division multiplexing (DWDM) networks as spare sources or fast wavelengthswitching devices.1,2 The wavelength monitor for controlling a tunable-laser diode must cope with multiple output wavelengths and the required tuning speed. The tuning curve of a TLS might change because of aging or variation in temperature. ${ }^{3}$ This change may give rise to mode hopping and incomplete tuning of a TLS. ${ }^{4}$ The most popular components for wavelength control of DWDM lasers are Fabry-Perot (FP) etalons because they are stable and compact. ${ }^{3-5}$ However, a FP etalon whose free spectral range (FSR) is usually chosen to be the same as the channel spacing cannot distinguish among different channels because it has a periodic wavelength. ${ }^{3}$ This makes it difficult to detect mode hopping and incomplete tuning. Etalons with special tuning mechanisms ${ }^{6,7}$

C.-L. Yang and S.-L. Lee (d8802304@mail.ntust.edu.tw) are with the Department of Electronic Engineering, National Taiwan University of Science and Technology, 43, Keelung Rd., Sec. 4, Taipei 106, Taiwan. J. Wu is with the Department of Electric Engineering, National Taiwan University, No. 1, Roosevelt Road, Section 4, Taipei 106, Taiwan.

Received 9 May 2003; revised manuscript received 4 November 2003; accepted 17 December 2003.

0003-6935/04/091914-08\$15.00/0

(C) 2004 Optical Society of America or array waveguide gratings $\mathrm{s}^{8,9}$ were also proposed for monitoring tunable lasers, but they either have limited response speed or require expensive components.

The monitoring of tunable DWDM lasers requires a wavelength-sensitive characteristic that has a steep slope about the wavelength grid to lock the wavelength precisely and that can distinguish among channels. The monitoring functions can be split into two parts, one for wavelength locking and the other for channel recognition. In the first path, the wavelength of a TLS can be locked to a DWDM wavelength grid specified by the International Telecommunication Union (ITU) by use of a FP etalon. The second path is used for resolving the channel position among different channels; this requires optoelectronic components with channel-dependent characteristics. Possible candidates include various optical filters, 3,10 tunable FP etalons, 6,7 arrayed waveguide gratings, $, 8,9$ Mach-Zehnder interferometers, ${ }^{11}$ long-period fiber gratings, ${ }^{12}$ and spectral filtering detectors. ${ }^{13,14}$

Here we propose to use the voltage induced across a semiconductor optoelectronic diode (SOD) for channel recognition. The SOD referred to here can be a FP laser diode (FPLD), a semiconductor optical amplifier (SOA), or a light-emitting diode (LED). Because the transparency of a SOD is wavelength dependent, the induced voltage is a function of wavelength. In principle, a FP laser diode alone can be used for both wavelength locking and channel recognition. With the help of cavity resonance, its 


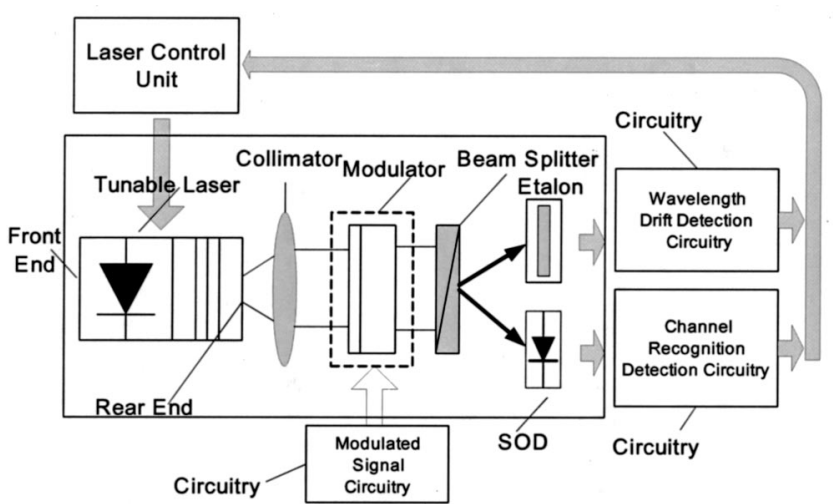

Fig. 1. Schematic of the proposed wavelength-control approach for a tunable laser.

voltage-versus-wavelength curve has a steep slope, which is good for wavelength locking. The voltage resonance is not truly periodic, however, and can be used to distinguish among channels of different wavelengths. A SOD for channel recognition is attractive because the wavelength sensor is compact and can be integrated monolithically with the tunable laser. However, to achieve satisfactory resolution for DWDM applications with a single FPLD requires precise temperature control, usually with better than a $0.01{ }^{\circ} \mathrm{C}$ margin of error, and highresolution scanning current. ${ }^{15}$

When the role of a SOD is for channel recognition only, the induced voltages between two adjacent channels are relatively far apart and can tolerate certain voltage fluctuations. Therefore the required temperature stability can be significantly relaxed. One can thus achieve a high-resolution monitoring technique by combining the advantages of the stable temperature properties of a FP etalon filter with the low cost and versatility of a SOD. One can easily tune a SOD to monitor different wavelength bands by adjusting its bias current. We describe and compare the performance of different types of SOD. The technique can also be applied in optical networking units for monitoring wavelength paths in a scanning mode.

\section{Principles of Operation}

Figure 1 is a schematic of the proposed technique of wavelength control of a tunable laser module. The laser output is first collimated and dithered at a specific frequency with an optical intensity modulator. The signal dithering is utilized to improve the monitoring sensitivity because it allows a narrowbandpass filter to be used to eliminate detector noise. For easy packaging with tunable lasers, modulators of simple structure and easy alignment, such as those made from liquid crystals ${ }^{16}$ or normal surface modulators, ${ }^{17}$ are particularly favored for such applications. The dithered signal is then divided into two paths, one to a FP etalon and the other to a SOD. As the TLS is tuned to output a given DWDM wavelength, the etalon must monitor the wavelength's de- viation from the wavelength grid and feed the deviation back to the laser control unit to adjust the wavelength to the closest wavelength grid. Wavelength accuracy better than $0.01 \mathrm{~nm}$ is usually specified for a commercial DWDM source that uses an etalon as the wavelength locker. Therefore the wavelength-locking path should be able to register the TLS wavelength at one of the wavelength grids with this degree of accuracy. Then the SOD will recognize the channel and detect whether mode hopping or incomplete tuning problems exist. If one of these problems does occur, the SOD unit must calculate the wavelength distance between the current channel and the expected channel and then instruct the control unit to adjust the wavelength further to the correct channel. This procedure will help the TLS control unit to make a tuning diagnosis because most TLSs suffer from drift in the tuning map as a result of aging or a change in ambient conditions. ${ }^{18,19}$

In addition to having a wavelength-locking function, a FP etalon can act as a precise wavelength reference because of its high resistance to temperature variation. One can achieve channel recognition, however, by detecting the induced junction voltage across the diode for a fixed bias current. Because the transparent current of a SOD varies with the incident wavelength, the voltage level in response to the dithered input power can indicate the wavelength if the bias is fixed. ${ }^{20}$ Although the detection of transparent current can also be used for channel recognition, detection of junction voltage is preferred here to simplify the detection circuit as well as to achieve rapid response. One can easily adjust the operation point of a SOD to match that of the etalon by tuning the bias current such that the two sensors can share the same temperature controller. Inasmuch as the SOD can easily be integrated with the TLS chip, the whole TLS module can still be packaged into a compact form. In the following sections the characteristics of the key components, including the FP etalon and SODs, are investigated and discussed.

\section{Wavelength Locking with a Fabry-Perot Etalon}

FSR and thermal stability are two critical parameters for a FP etalon that is used as a wavelength locker. The channel spacing of the monitored DWDM TLS is usually an integer multiple of the FSR such that one etalon can monitor many ITU channels. In our experiments the FSR is chosen to be $50 \mathrm{GHz}$, which is equal to half of the channel spacing of the tunable laser. This selection doubles the sensitivity to wavelength drift by means of a compromise in the wavelength-locking range.

The thermal characteristic of the etalon must be a compromise between stability and agility. The etalon needs to be thermally tuned to align multiple ITU wavelengths at intersection points of the transmitted and reflected curves. As shown in Fig. 2(a), the intersection point varies at a rate of $0.011 \mathrm{~nm} /{ }^{\circ} \mathrm{C}$. If the tolerance on the wavelength deviation is $0.01 \mathrm{~nm}$, this etalon can tolerate as much as a $0.9{ }^{\circ} \mathrm{C}$ temper- 

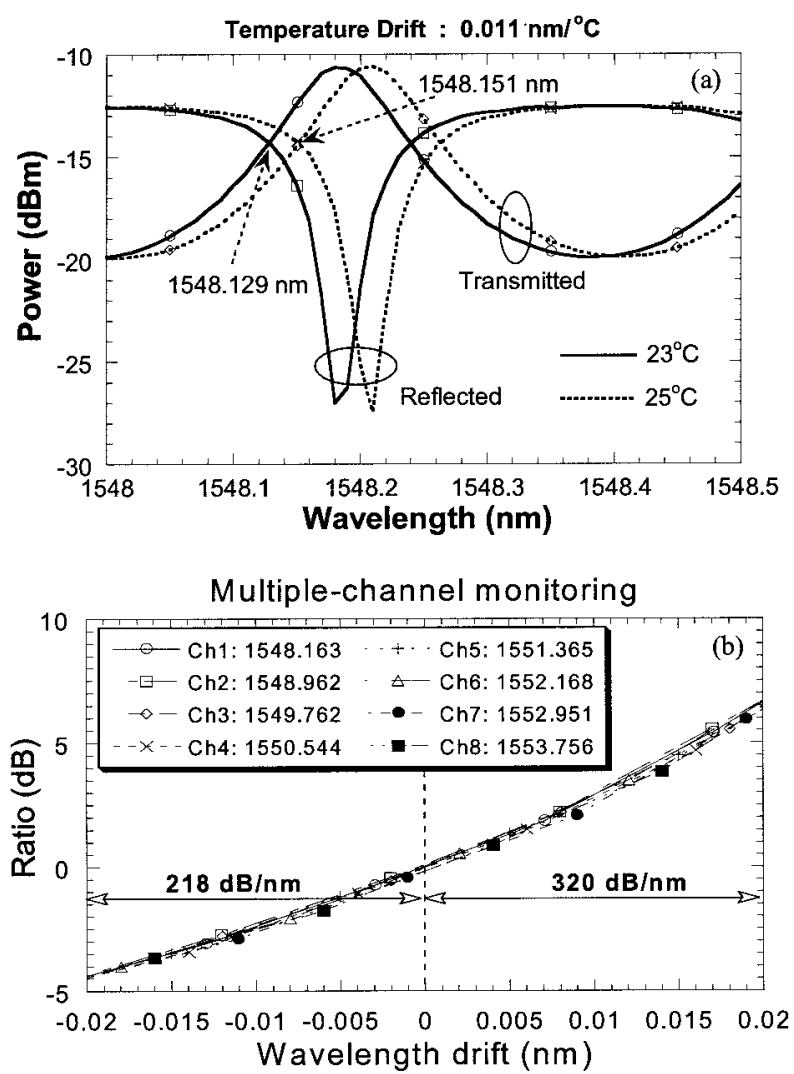

Fig. 2. Characteristics of the FP etalon: (a) temperature variation of the locking point and (b) ratio of the transmitted signal to the reflected signal for eight ITU wavelengths.

ature change. To align the etalon to an ITU grid, however, needs a wavelength tuning of at most half of the etalon FSR (0.2 $\mathrm{nm}$ for our experiments), which needs a maximal temperature change of $\sim 18^{\circ} \mathrm{C}$. Because temperature control is required in our approach to stabilizing the SOD sensor, the etalon can share the same temperature control. Thus an etalon of lower thermal expansion is not needed.

In our experiments the intersection point of the transmitted and reflected curves is used as the monitoring point. Figure 2(b) shows the responses of the etalon to eight wavelengths of $100-\mathrm{GHz}$ channel spacing at the same temperature, $26^{\circ} \mathrm{C}$. As the channel spacing is twice the FSR, the ratio of voltage to wavelength deviation is almost identical among the channels. Therefore the monitoring circuit needs to memorize only one of these curves. The maximal fluctuation among the curves results in a wavelength deviation of $\sim 0.005 \mathrm{~nm}$, which is satisfactory for 50 or 100-GHz-spaced channels.

\section{Channel Recognition with Semiconductor Optical Diodes}

We classify the SODs into two categories, resonant and nonresonant devices, as illustrated in Fig. 3. The former includes most types of laser diode, and the latter includes SOAs and LED's. The gain material for these active components has a transparent current that decreases as the wavelength moves to the

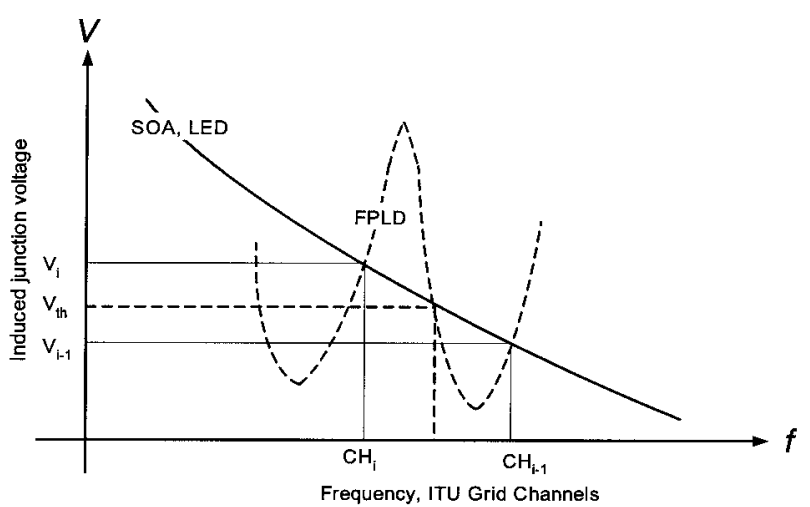

Fig. 3. Illustration of the induced junction voltage for resonant and nonresonant SODs. The decision threshold $\mathrm{V}_{\mathrm{th}}$ is also shown.

long-wavelength side. Acting as a wavelength sensor, a SOD must be biased below threshold. Under such conditions the optical property inside a SOD is dominated by spontaneous emission when there is no input light. It becomes abundant in stimulated emission when sufficient light whose wavelength falls within the gain spectrum is injected upon the diode. Thus the dithered incident light causes carrier modulation and results in voltage modulation across the diode junction. This voltage is a function of the incident wavelength. We have addressed the operation principles of wavelength sensing, using the transparent properties of a SOA. ${ }^{20}$ High-resolution wavelength discrimination by differential detection of FPLD voltage was demonstrated. ${ }^{15}$ As the requirements for the SOD characteristics for applications as wavelength lockers are different from those for channel recognizers, we discuss in detail the SOD characteristics for channel recognition. The performance of resonant and nonresonant types of SODs are compared.

For use as a wavelength sensor, the facet reflection of a FPLD causes a variation in the resonance of the induced voltage that is related to the wavelength. The channel number can be resolved by comparison of the induced voltage and a threshold voltage $\left(V_{\text {th }}\right)$, as indicated in Fig. 3. Figure 4 shows the induced voltage relative to frequency and temperature for a 1.55- $\mu \mathrm{m}$ FPLD. The wavelength spacing between the resonant peaks, i.e., a 97-GHz FSR, is equal to the cavity mode spacing of the FPLD. Therefore the FPLD must be carefully cleaved to match the cavity mode spacing to the DWDM channel spacing. For example, $1 \%$ error in the cleavage position results in $1 \mathrm{GHz}$ of error in the cavity mode spacing for a FSR of $100 \mathrm{GHz}$. This corresponds to a cleavage error of $4 \mu \mathrm{m}$ for a $400-\mu \mathrm{m}$ long FPLD. The required cleavage accuracy can be achieved by use of a standard laser cleavage tool.

The error in the FSR of a FPLD will limit the number of channels that can be monitored by a FPLD. The number is determined by the ratio of locking range to error. The locking range for the curves shown in Fig. 4 is $\sim 0.25 \mathrm{~nm}$ (or $31 \mathrm{GHz}$ ) for the 


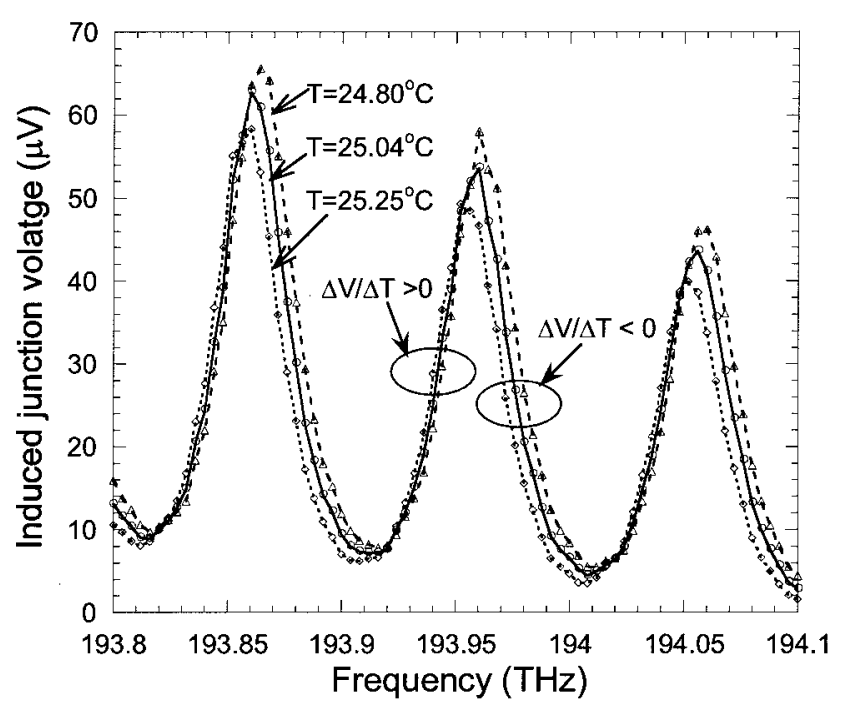

Fig. 4. Temperature variation of the induced junction voltage for a FPLD.

positive slope, and the error in the FSR is $3 \mathrm{GHz}$. Thus this FPLD can monitor $\sim 11$ channels. The locking range is larger for aligning the channel position at the negative slope. Note that the data shown in Fig. 4 are measured from a commercial FPLD that has not been specially made for channel sensing applications. If the FPLD is carefully cleaved to have less than $4 \mu \mathrm{m}$ of error, it can monitor $\sim 30$ channels.

Thermal stability depends also on where the channels are aligned to the curve. From Fig. 4, if the monitored channel is aligned to the positive slope of the induced voltage curve the voltage increases as the temperature rises. The voltage decreases with increasing temperature, however, as the channel is aligned at the negative slope. To achieve better thermal stability and wavelength discrimination, one usually chooses the monitoring point at the middle of positive slope, which has a smaller thermal change rate.

Ideally, the transparent properties of a SOD should be free from the influence of facet resonance. However, because of amplified spontaneous emission (ASE) the carrier density is nonuniform along the longitudinal direction. The measured transparent current is an average over the laser length, so the detected voltage for a given bias current is the average induced voltage across the cavity. The incident light affects the carrier distribution along the SOD, so the voltage depends on the optical power distribution in the cavity. This causes the induced voltage of a FPLD to be resonant and gives rise to a higher resolution at the middle of slope than in nonresonant diodes.

A SOD can provide gain (loss) to the incident light if the input wavelength is larger (smaller) than the corresponding transparent current for the bias current. The bias current of a SOD can be quickly tuned to monitor different wavelength bands. For a FPLD sensor, additional fine tuning is required for aligning the monitored channel to the middle of the positive slope. The resonance is stronger in the gain regime. The operation of a nonresonant SOD is simpler and can be achieved in both regimes. The monitored wavelength range for a given bias is larger when a phase-sensitive detector, such as a lock-in amplifier, is used because the responses for the gain and loss regimes can be distinguished.

\section{Wavelength Sensitivity of Semiconductor Optical Diodes}

The induced junction voltage across a SOD is linearly proportional to the average optical power inside the diode. The average photon density and the effects of ASE on the characteristics of SOAs were addressed in Ref. 21. As the facet resonance and the ASE are included, the voltage for a SOD can be approximated in the form

$$
V=\eta_{V}\left(I-I_{\mathrm{tr}}\right)\left\{\frac{P_{\mathrm{in}} G_{s}}{1+\gamma \sin ^{2}\left[\pi\left(f-f_{r} / \nu_{\mathrm{FSR}}\right)\right]}\right\}+V_{\mathrm{ASE}},
$$

where $\eta_{V}$ is a conversion factor, $I_{\mathrm{tr}}$ is the transparent current; $P_{\text {in }}$ is the incident optical power, and $\nu_{\mathrm{FSR}}$ and $f_{r}$ are the FSR and a reference frequency for a resonant $\mathrm{SOD}$, respectively. $V_{\mathrm{ASE}}$ is the voltage offset that is due to the ASE and the source's spontaneous-emission noise. The $\gamma$ factor indicates the resonance strength and is zero for a SOA with perfect antireflection coatings. $G_{s}=\exp \left[-\alpha\left(f-f_{r}\right)\right]$ is the single-pass gain. Figure 5 shows that curve fitting with Eq. (1) matches well the measured data for a FPLD and a SOA. The input power for both measurements is $P_{\text {in }}=0.316 \mathrm{~mW}$. The fitting parameters for the FPLD are $\nu_{\mathrm{FSR}}=97 \mathrm{GHz}, \eta_{V}=$ $63.29 \mu \mathrm{V} \mathrm{mA}{ }^{-1} \mathrm{~mW}^{-1}, \alpha=2.78 \times 10^{-13} \mathrm{~Hz}^{-1}, \gamma=4$, $f_{r}=195057.5 \mathrm{GHz}$, and $V_{\mathrm{ASE}}=-3 \mu \mathrm{V}$ with a bias current of $I=16.5 \mathrm{~mA}$. For the SOA the fitting parameters are $\eta_{V}=12.658 \mu \mathrm{V} \mathrm{mA}{ }^{-1} \mathrm{~mW}^{-1}, \alpha=$ $2.22 \times 10^{-13} \mathrm{~Hz}^{-1}, \gamma=0, f_{r}=194240 \mathrm{GHz}$, and $V_{\mathrm{ASE}}=0$ with a bias current of $I=29.2 \mathrm{~mA}$. Because $\gamma$ is zero, the curve for the SOA is monotonic. Note that the FPLD has approximately five times larger $\eta_{V}$ than the SOA. Although $\eta_{V}$ depends also on the material parameters of the SOD, the larger $\eta_{V}$ of the FPLD is due mainly to the increase in the average photon density inside the cavity as a result of the resonant effect.

It is clear from Fig. 4 that resonant effects can enhance the local wavelength resolution if the channel is aligned to the middle of a slope in the voltageversus-frequency (or -wavelength) curve. Thus a FPLD can be used for wavelength sensing with high resolution if the requirement for precise temperature control can be overcome. For applications in channel recognition, the channel-to-channel variation of induced voltage is of major concern. For SOAs the 

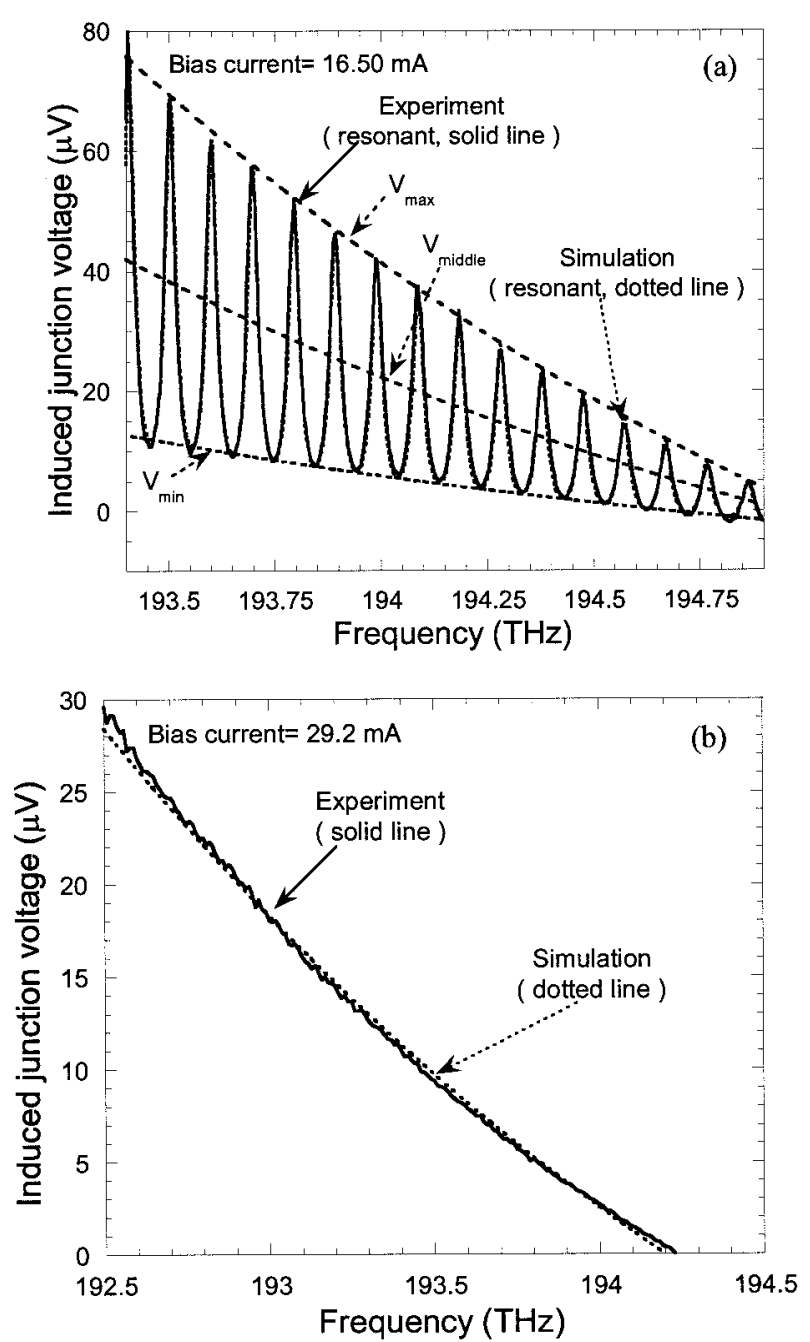

Fig. 5. Curve fitting to match the measured data for (a) a FPLD and (b) a SOA.

induced voltage change between the adjacent channels is

$$
\Delta V \approx \eta_{V} P_{\text {in }}\left|\frac{\partial G_{s}\left(I-I_{\mathrm{tr}}\right)}{\partial \lambda}\right| \Delta \lambda,
$$

where $\Delta \lambda$ is the channel spacing. For a FPLD the voltage changes for two special conditions are considered. The first occurs when all the monitored channels are aligned to the resonant peak, i.e., when $f-$ $f_{r}=m v_{\mathrm{FSR}}$, where $m$ is an integer. Under this condition the channel-to-channel change in the induced voltage can be expressed in the same form as Eq. (2) but with a larger $\eta_{V}$. The other condition occurs at the middle of the slope, where $f-f_{r} \cong(m+1 / 7) \nu_{\mathrm{FSR}}$. In this condition the local change rate of the induced voltage reach its maximum, while the voltage change between two adjacent channels is approximately

$$
\Delta V \approx 0.6 \eta_{V} P_{\text {in }}\left|\frac{\partial G_{s}\left(I-I_{\text {tr }}\right)}{\partial \lambda}\right| \Delta \lambda
$$

$V_{\mathrm{ASE}}$ is neglected in Eqs. (2) and (3) because the major effect of the ASE and the source's spontaneous emission on the induced voltage is the shift in the zero-voltage frequency. ${ }^{22}$ Despite the fact that $V_{\mathrm{ASE}}$ is slightly wavelength dependent, spontaneous emission has a negligible effect on the slope of the voltageversus-frequency curve because of the relative small voltage effect. Therefore the channel-to-channel wavelength sensitivity can be expressed as

$$
\frac{\partial V}{\partial \lambda}=b \eta_{V}\left(I-I_{\mathrm{tr}}\right) P_{\mathrm{in}} \frac{\partial G_{s}}{\partial \lambda}-b \eta_{V} G_{s} P_{\mathrm{in}} \frac{\partial I_{\mathrm{tr}}}{\partial \lambda},
$$

where $b=1$ for SOAs and FPLDs with peak detection, whereas $b=0.6$ for monitoring by use of the middle of the slope. The transparent current of a gain material varies with wavelength in the form ${ }^{20}$

$$
\frac{\partial I_{\mathrm{tr}}}{\partial \lambda}=-\frac{E}{\xi k T} \frac{I_{\mathrm{tr}}}{\lambda},
$$

where $E$ is the photon energy that is inversely proportional to $\lambda, \xi$ is the ideality factor for the wavelength dependency, and $k T$ is the thermal energy. Thus Eq. (4) can be rewritten as

$$
\frac{\partial V}{\partial \lambda}=\frac{b \eta_{V} G_{s} P_{\mathrm{in}}}{\lambda}\left[c_{1}\left(I-I_{\mathrm{tr}}\right)+c_{2} I_{\mathrm{tr}}\right]
$$

where $c_{1}=\alpha f$ and $c_{2}=E / \xi k T$. The two terms in brackets in Eq. (6) have the same sign when the SOD is biased at the gain regime $\left(I>I_{\mathrm{tr}}\right)$, and they have opposite sign in the loss regime. The values of $c_{1}$ and $c_{2}$ are comparable. Therefore the induced voltage can have a larger slope relative to wavelength in the gain regime than in the loss regime, although the latter case usually has a larger differential gain.

The resonance of a FPLD enhances the signal level. In the ideal case, the voltage change between two adjacent channels is maximal when all channels are aligned to the resonant peaks of a FPLD. Moreover, one can recognize the channels by locating the voltage peak where the signal-to-noise ratio is better. However, to do so requires the channel spacing to be exactly equal to the cavity mode spacing of the FPLD to permit multiple DWDM channels to be monitored. The $V$-versus- $\lambda$ curve for a nonresonant SOD is monotonic, however, so the control circuitry can be simpler and a larger number of channels can be monitored.

\section{Demonstration and Discussion}

In the proposed method the output wavelength of a tunable laser is locked to a DWDM channel by use of a FP etalon, so the channel recognizer does not need high wavelength resolution. The decision threshold is usually placed at the intersection of the responses for two adjacent channels, as indicated in Fig. 3. Therefore a larger tolerance of wavelength or temperature variation can be achieved.

To demonstrate the feasibility of our tunablewavelength controller we used a commercial fiberpigtailed FPLD with a cavity mode spacing of 97 
Table 1. Induced FPLD Voltages for Eight Channels and Voltage Deviations for Wavelength or Temperature Drift

\begin{tabular}{|c|c|c|c|c|c|c|c|c|}
\hline & Channel 1 & Channel 2 & Channel 3 & Channel 4 & Channel 5 & Channel 6 & Channel 7 & Channel 8 \\
\hline Channel Frequency $(\mathrm{THz})$ & 193.4 & 193.5 & 193.6 & 193.7 & 193.8 & 193.9 & 194.0 & 194.1 \\
\hline Induced voltage $(\mu \mathrm{V})$ & 32.2 & 25.3 & 19.7 & 15.4 & 11.1 & 7.8 & 5.1 & 2.4 \\
\hline Wavelength slope $(\mu \mathrm{V} / \mathrm{nm})$ & 191.9 & 129.9 & 112.8 & 92.9 & 37.4 & 34.5 & 33.8 & 1.5 \\
\hline $\begin{array}{l}\Delta V \text { for } \Delta \lambda= \pm 0.02 \mathrm{~nm} \\
\quad(\mu \mathrm{V})\end{array}$ & \pm 3.9 & \pm 2.6 & \pm 2.3 & \pm 1.9 & \pm 0.8 & \pm 0.7 & \pm 0.7 & \pm 0.04 \\
\hline Temperature slope $\left(\mu \mathrm{V} /{ }^{\circ} \mathrm{C}\right)$ & -23.5 & -18.4 & -15.8 & -12.1 & -8.8 & -8.0 & -6.0 & -4.3 \\
\hline$\Delta V$ for $\Delta T= \pm 0.15{ }^{\circ} \mathrm{C}(\mu \mathrm{V})$ & \pm 3.5 & \pm 2.8 & \pm 2.3 & \pm 1.8 & \pm 1.3 & \pm 1.2 & \pm 0.9 & \pm 0.7 \\
\hline
\end{tabular}

GHz. A packaged FPLD was used for obtaining stable and repeatable results. If the FPLD were specially cleaved for applications as a channel sensor, its cavity mode spacing should be close to $100 \mathrm{GHz}$, the channel spacing. Its frequency-dependent voltage characteristic is shown in Fig. 4. Inasmuch as the 97-GHz cavity mode spacing is slightly less than the $100-\mathrm{GHz}$ channel spacing, the channels are aligned to the negative slope $\left(I=15.7 \mathrm{~mA}, T=25.04{ }^{\circ} \mathrm{C}\right)$. The induced junction voltage of the negative slope is more sensitive to temperature variation than that of the positive slope. The results are summarized in Table 1. To minimize the temperature variation for operation at the negative slope, the channels are allocated at the bottom half of the slope. That is, the first channel is aligned near the bottom of the negative slope and the last channel is operated toward the middle of the negative slope. Thus the lowerfrequency channel has larger sensitivity in the induced voltage for wavelength drift and temperature variation. Though the voltage difference is not constant for all channels, each channel has nearly the same wavelength tolerance, $\pm 0.15^{\circ} \mathrm{C}$, and wavelength tolerance, $0.02 \mathrm{~nm}$. The wavelength tolerance is relatively easy to achieve with the FP etalon.

When a nonresonant component such as a SOA is used as the wavelength sensor, its monotonic $V$-versus- $\lambda$ curve simplifies the procedure for channel recognition. The induced voltage is relatively insensitive to wavelength change, but $100-\mathrm{GHz}$ channels can still be clearly resolved from the voltage. Operating the sensor at the gain regime can produce an induced junction voltage that is more sensitive to frequency change than operation in the loss regime, but the former condition is also more sensitive to the residual cavity resonance. If the phase-sensitive detector is used to obtain the induced voltage, the signals for operating at the gain regime and the loss regime can be distinguished from each other. The junction voltage induced in response to the wavelength and temperature drift for eight channels has nearly the same slope, as listed in Table 2.

The wavelength tolerance of channel recognition is relaxed to half of the channel spacing for a nonresonant sensor. For instance, the temperature stability must be kept within $0.006{ }^{\circ} \mathrm{C}$ to produce a wavelength stability of $0.01 \mathrm{~nm}$ for using a $1.55-\mu \mathrm{m}$ SOA as a wavelength sensor. ${ }^{20}$ The temperature tolerance increases to $\pm 0.25^{\circ} \mathrm{C}$, as shown in Table 2 , when the SOA is used for channel recognition for a tunable laser of $100-\mathrm{GHz}$ channel spacing. For comparison, the temperature tolerance for using a FPLD in the same applications is smaller $\left( \pm 0.15^{\circ} \mathrm{C}\right)$ because of the resonant effect.

Finally, in Fig. 6 we demonstrate tunablewavelength monitoring with a combination of a FP etalon and a FPLD. An external-cavity tunable laser was tuned to eight DWDM channels in $100-\mathrm{GHz}$ steps. The junction voltage of the FPLD changes in a staircase form in response to wavelength tuning. A tiny signal appears at the output of the etalon circuit because the wavelengths are allocated at the wavelength grid. The etalon signal indicates a maximal wavelength deviation of $0.008 \mathrm{~nm}$. The wavelength tuning unit of a tunable laser can use the etalon signal to adjust the wavelength further with better accuracy. As the channel wavelength falls within the locking range, the channel number can be clearly recognized from the induced voltage. When a SOA is used and the tunable laser is tuned over 35 channels, Fig. 7 shows that the channel wavelengths can be clearly resolved from the junction voltage. In the experiments the SOA is biased at the transparent current for the 1548.134-nm channel. Phasesensitive detection is employed to yield a voltage such that the channels that reside in the gain regime of the

Table 2. Induced SOA Voltages for Eight Channels and Voltage Deviations for Wavelength or Temperature Drift

\begin{tabular}{lcccccccc}
\hline & Channel 1 & Channel 2 & Channel 3 & Channel 4 & Channel 5 & Channel 6 & Channel 7 & Channel 8 \\
\hline Channel frequency $(\mathrm{THz})$ & 193.7 & 193.8 & 193.9 & 194 & 194.1 & 194.2 & 194.3 & 194.4 \\
Induced voltage $(\mu \mathrm{V})$ & 14.2 & 12.3 & 10.7 & 9.3 & 7.6 & 6.2 & 4.9 & 3.7 \\
Wavelength slope $(\mu \mathrm{V} / \mathrm{nm})$ & 4.1 & 3.8 & 3.7 & 3.3 & 1.8 & 2.3 & 2.0 & 1.9 \\
$\Delta V$ for $\Delta \lambda= \pm 0.03 \mathrm{~nm}(\mu \mathrm{V})$ & \pm 0.13 & \pm 0.12 & \pm 0.11 & \pm 0.11 & \pm 0.07 & \pm 0.06 & \pm 0.06 & \pm 0.06 \\
Temperature slope $\left(\mu \mathrm{V} /{ }^{\circ} \mathrm{C}\right)$ & -3.2 & -3.1 & -3.0 & -2.8 & -2.5 & -2.5 & -2.1 & -2.2 \\
$\Delta V$ for $\Delta T= \pm 0.25^{\circ} \mathrm{C}(\mu \mathrm{V})$ & \pm 0.8 & \pm 0.8 & \pm 0.7 & \pm 0.7 & \pm 0.6 & \pm 0.6 & \pm 0.5 & \pm 0.5 \\
\hline
\end{tabular}




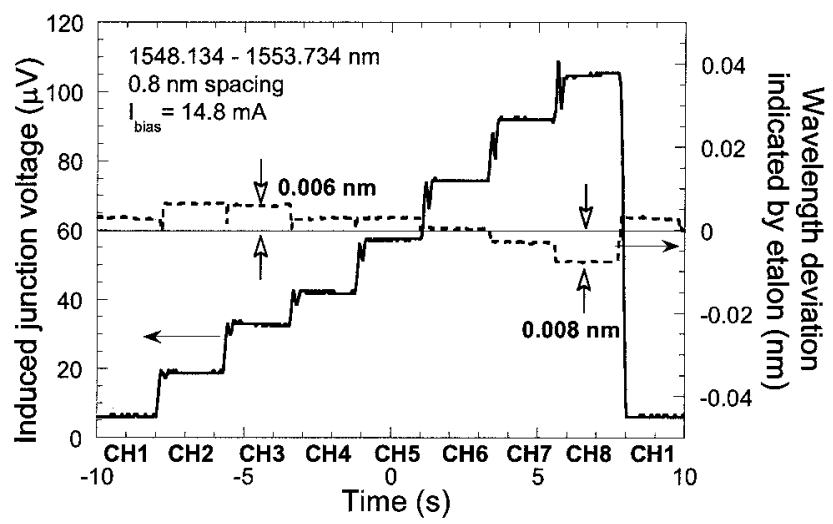

Fig. 6. Tunable monitoring of eight $100-\mathrm{GHz}$-spaced channels with a FPLD and a FP etalon. The light is modulated at $1 \mathrm{kHz}$.

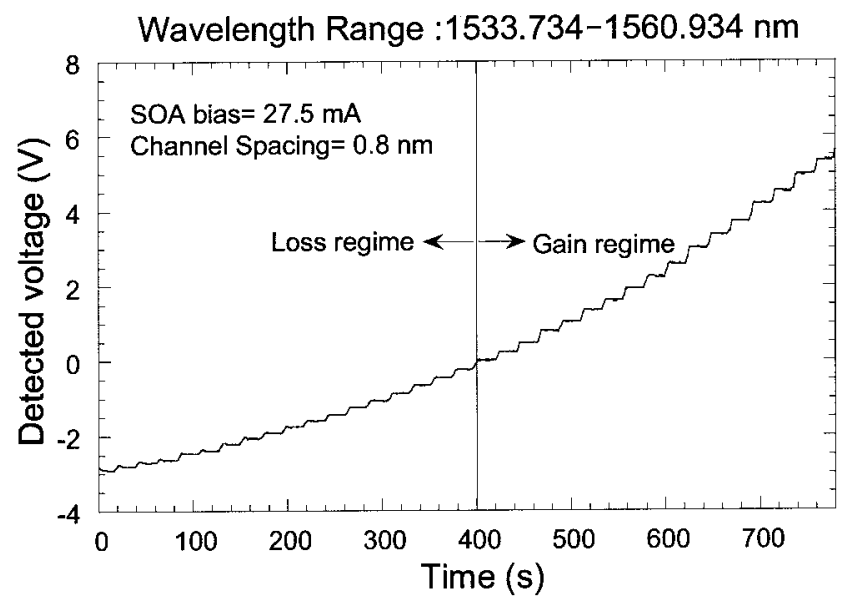

Fig. 7. Output voltage of a SOA for 35 channels of $100-\mathrm{GHz}$ spacing. The induced voltage was amplified by $2 \times 10^{5}$.

SOA can be distinguished from those in the loss regime. Note that, as a nonresonant SOD is used, the channel positions can be determined from the junction voltage without the need to wait for the etalon to bring the wavelength to the closest wavelength grid.

\section{Comparison and Conclusions}

Among various types of SOD used for monitoring DWDM tunable lasers in the $1.55-\mu \mathrm{m}$ wavelength band, a FPLD may currently offer the solution that is easiest to obtain at lowest cost. This is so because of the large volume of FPLDs used as light sources for transmitting signals over optical fibers. The resonant effect of a FPLD can also enhance the induced junction voltage, but this requires relatively precise control in the operation condition. With a $1 \%$ error tolerance on cleavage position, a FPLD can monitor $\sim 30,100-\mathrm{GHz}$ spaced channels. Monitoring a widely tunable laser that can output much more channels requires a nonresonant sensor with monotonic wavelength variation of junction voltage. A SOA for this application can be inexpensive because it does not require precise antireflection coating as is required for conventional optical amplifier applications. The other choice is an edge-emitting LED (ELED). The typical structure of an ELED in a 1.3- or 1.55- $\mu \mathrm{m}$ wavelength band requires antireflection coating and an absorber at one end to eliminate the resonant effect. An ELED needs more fabrication steps than a FPLD, so its price falls between those of a SOA and a FPLD. In principle, a SOA for wavelength-sensing applications does not need two-end optical coupling, whereas an ELED for this application requires temperature control. A surface-emitting LED has a relatively simple fabrication procedure and is geometrically symmetric, which gives rise to polarization-insensitive induced junction voltage. However, a surface-emitting LED at the $1.55-\mu \mathrm{m}$ wavelength band is relatively hard to obtain in the current commercial market because it is not the favorite choice as a signal source for optical communications. This situation should be reversed after the application of a surface-emitting LED as a wavelength sensor becomes widespread.

In summary, we have proposed and demonstrated a wavelength-control technique for tunable lasers by combining the frequently used FP etalon with a SOD. The etalon provides stable wavelength locking, and the SOD performs channel recognition to detect problems of mode hopping and incomplete tuning. These components can be integrated into a compact module to further reduce cost and improve reliability. The approach can have a fast response speed, so it can be used to monitor the fast tunable lasers that are key devices for optical burst switching and optical packet switching. The required temperature control is relaxed to a practical range because the SOD is simply used for channel recognition.

This research was supported in part by the Computer \& Communications Research Laboratory, Industrial Technology Research Institute, Taiwan, under grant T2-90065, and by the Ministry of Education, Taiwan, under grant 89-E-FA06-2-4.

\section{References}

1. F. Delorme, "Widely tunable 1.55- $\mu \mathrm{m}$ lasers for wavelengthdivision-multiplexed optical fiber communications," IEEE J. Quantum Electron. 34, 1706-1716 (1998).

2. D. J. Blumenthal, J. E. Bowers, L. Rau, H.-F. Chou, S. Rangarajan, W. Wang, and H. N. Poulsen, "Optical signal processing for optical packet switching networks," IEEE Commun. Mag. 41(2), 23-29 (2003).

3. D. Anthon, J. Berger, K. Cheung, A. Fennema, S. Hrinya, H. Lee, and A. Tselikov, "Frequency and mode control of tunable external cavity semiconductor lasers," in Optical Fiber Communication Conference, Vol. 86 of OSA Trends in Optics and Photonics Series (Optical Society of America, Washington, D.C., 2003), Vol. 1, pp. 74-75.

4. D. A. Ackerman, K. F. Dreyer, U. Koren, J. W. Stayt, S. L. Broutin, W. A. Asous, J. E. Johnson, L. J.-P. Ketelsen, K. K. Kamath, S. O'Brien, W. J. Shakespeare, M. A. Eshelman, M. M. Meyers, D. A. Snyder, and E. S. Mak, "Wavelength, modal, and power stabilization of tunable electro-absorption modulated distributed Bragg reflector lasers," presented at the International Semiconductor Laser Conference, Monterey, Calif., 26 Sept. 2000.

5. M. Imaki, Y. Mikami, M. Sato, Y. Nishimura, A. Adachi, and Y. 
Hirano, "Athermal birefringent solid etalon for $25 \mathrm{GHz}$ spacing built-in wavelength monitor," in Optical Fiber Communication Conference, Vol. 86 of OSA Trends in Optics and Photonics Series (Optical Society of America, Washington, D.C., 2003), Vol. 2, pp. 762-763.

6. C. M. Miller and J. W. Miller, "Wavelength-locked, two-stage fibre Fabry-Perot filter for dense wavelength division demultiplexing in erbium-doped fibre amplifier spectrum," Electron. Lett. 28, 216-217 (1992).

7. T. Niemi, M. Uusimmaa, S. Tammela, P. Heimalaia, T. Kajava, and H. Ludvigsen, "Tunable silicon etalon for simultaneous spectral filtering and wavelength monitoring of a DWDM transmitter," IEEE Photon. Technol. Lett. 13, 58-60 (2001).

8. K. R. Tamura, Y. Inoue, K. Sato, T. Komukai, A. Sugita, and M. Nakazawa, "32 wavelength tunable mode-locked laser with $100-\mathrm{GHz}$ channel spacing using an arrayed waveguide grating," in Optical Fiber Communication Conference, Vol. 7 of OSA Trends in Optics and Photonics Series (Optical Society of America, Washington, D.C., 2001), paper TuJ5.

9. H. Li, S. Zhong, X. Yang, Y. J. Chen, and D. Stone, "Full coverage multichannel wavelength monitoring circuit using centre-offset phased-array waveguide grating," Electron. Lett. 34, 2149-2151 (1998).

10. M. Imaki, S. Yamamoto, M. Sato, Y. Nishimura, K. Masuda, S. Takagi, A. Adachi, J. Yamashita, and Y. Hirano, "Wideband athermal wavelength monitor integrated wavelength temperature-tunable DFB-LD module," Electron. Lett. 37, 1035-1036 (2001).

11. H. H. Yaffe, C. H. Henry, R. F. Kazarinov, and M. A. Milbrodt, "Polarization-independent silica-on-silicon Mach-Zehnder interferometers," J. Lightwave Technol. 12, 64-67 (1994).

12. A. M. Vengsarkar, P. J. Lemaire, J. B. Judkins, V. Bhatia, T. Erogan, and J. E. Sipe, "Long-period fiber gratings as bandrejection filters," J. Lightwave Technol. 14, 58-65 (1996).
13. T. Coroy, R. M. Measures, T. H. Wood, and C. A. Burrus, "Active wavelength measurement system using an InGaAsInP quantum-well electroabsorption filtering detector," IEEE Photon. Technol. Lett. 8, 1686-1688 (1996).

14. A. Densmore and P. E. Jessop, "A quantum-well waveguide photodetector for high-precision wavelength monitoring about $1.55 \mu \mathrm{m}$," IEEE Photon. Technol. Lett. 11, 1653-1655 (1999).

15. S.-L. Lee, Y.-Y. Hsu, and C.-T. Pien, "High-resolution wavelength monitoring using differential/ratio detection of junction voltage across a diode laser," IEEE Photon. Technol. Lett. 13, 872-874 (2001).

16. B. E. A. Saleh and M. C. Teich, Fundamentals of Photonics (Wiley, New York, 1991), pp. 724-726.

17. K. W. Goossen, J. A. Walker, and S. C. Arney, "Silicon modulator based on mechanically-active anti-reflection layer with 1 Mbit/sec capability for fiber-in-the-loop applications," IEEE Photon. Technol. Lett. 6, 1119-1121 (1994).

18. G. Sarlet, G. Morthier, and R. Baets, "Control of widely tunable SSG-DBR lasers for dense wavelength division multiplexing," J. Lightwave Technol. 18, 1128-1138 (2000).

19. H. Ishii, H. Yasaka, H. Tanobe, and Y. Yoshikuni, "Wavelength stabilization of a three-electrode distributed Bragg reflector laser with longitudinal mode control," Electron. Lett. 33, 494-496 (1997).

20. S.-L. Lee, C.-T. Pien, and Y.-Y. Hsu, "Operation principles of wavelength sensing using transparent properties of semiconductor optical diodes," J. Lightwave Technol. 19, 655-665 (2001).

21. M. J. Adams, J. V. Collins, and I. D. Henning, "Analysis of semiconductor laser optical amplifiers," IEE Proc. J. 132(1), 58-63 (1985).

22. G. E. Shtengel and D. A. Ackerman, "Internal optical loss measurements in $1.3 \mu \mathrm{m}$ InGaAsP lasers," Electron. Lett. 31, 1157-1159 (1995). 